\title{
Correlation of Pap smear and colposcopic finding with directed biopsy in detection of cervical neoplasm
}

\author{
Upadhyay $\mathbf{J}^{1}$, Garg $\mathrm{S}^{2}$ \\ ${ }^{1}$ Dr. Jigna Upadhyay, Associate Professor, ${ }^{2}$ Dr. Sameep Garg, Assistant Professor, both authors are affiliated with \\ Department of Pathology, Gujarat Adani Institute of Medical Science, Bhuj, Kutch, Gujarat, India.
}

Address for Correspondence: Dr. Sameep Garg, Department of Pathology, Gujarat Adani Institute of Medical Science, Bhuj, Kutch, Gujarat. E-mail: researchguide86@gmail.com,drpiyushpujara@gmail.com

\begin{abstract}
Introduction: Cancer of uterine cervix is the most common genital tract malignancy. Pap smear though widely used screening test for cervical cancer has the disadvantage of low sensitivity. Colposcopy has higher sensitivity compared to Pap smear. Concurrent screening with Pap smear and colposcopy can overcome this problem. Aim: The aim of the study was correlation of Pap smear and colposcopic finding with directed biopsy in detection of cervical neoplasm. Methods: The Present study was conducted in the Department of Pathology, Gujarat Adani Institute of Medical Science, Bhuj, Kutch. During the study period in 57 symptomatic patient's simultaneous Pap smear, colposcopic examination, followed by directed biopsy was performed. Data was recorded and analyzed. Results: In correlation between cytology and biopsy, sensitivity of cytology was $82.3 \%$, specificity $96.9 \%$ and accuracy of $92 \%$. In correlation between colposcopy and biopsy, sensitivity of colposcopy was $94.1 \%$, specificity $87.8 \%$ and accuracy of $90 \%$. Conclusion: High sensitivity in colposcopy as compared to cytology and high specificity in cytology as compared to colposcopy emphasizes the need for pairing these methods to achieve better results.
\end{abstract}

Keywords: Accuracy, Cancer, Colposcopy, cytology, Histopathology, Pap smear

\section{Introduction}

Cancer of cervix is the leading cause of cancer-related death among women in developing countries, where more than $80 \%$ of new cases occur. Cervical cancer continues to be the most common genital tract malignancy in India. Cancer of cervix is preceded by recognizable precancerous histological and cytological changes which provide opportunity for early detection of cervical neoplasm $[1,2]$.

Cervical cytology is the accepted method of screening for cervical cancer all over the world but it has low sensitivity. In the presence of an abnormal Pap smear, a tissue diagnosis is essential before proceeding with definitive therapy. Although cytology is accurate in predicting severity of cervical lesion, it cannot determine their location or extent. A random cervical biopsy in the absence of visible lesion may result in a false negative histologic diagnosis [3].

Manuscript received: $30^{\text {th }}$ October 2017

Reviewed: $8^{\text {th }}$ November 2017

Author Corrected: $17^{\mathrm{th}}$ November 2017

Accepted for Publication: $23^{\text {rd }}$ November 2017
With the introduction of colposcope, comparative studies substantiated that it was possible to accurately localize the area of abnormal cervical epithelium by colposcopic examination for the selection of biopsy site. Colposcopy as an adjunctive screening test has high sensitivity and can provide immediate results for evaluation of cervical lesions. Executing targeted biopsy, colposcopy can be useful in defining diagnosis of preinvasive lesions and carcinoma of cervix [4,5].

Complete and accurate assessment of the nature of a cervical neoplastic lesion relies on three methods of investigation: cervical smear, examination of cervix with colposcope and histology of a biopsy specimen.

Ideally the grade of cervical neoplasia discovered during all three methods should be the same, but in practice disagreement of more than one method is not uncommon [6]. The aim of the study was correlation of Pap smear and colposcopic finding with directed biopsy to assess the advantage of concurrent testing by 
cytology and colposcopy in the detection of cervical neoplasm.

\section{Materials and Methods}

Study Design and Study Settings- The Present study was conducted in the Department of Pathology, Gujarat Adani Institute of Medical Science, Bhuj, Kutch for a period of 12 months after taking approval from Institutional Ethical Committee.

Sample Collection- During the study period 57 female patients above the age of 18 years, with symptoms of vaginal discharge and other gynecological problems attending the outpatient department of OBG following informed consent were subjected for concurrent Pap smear examination, colposcopy and directed biopsy.

Inclusion and Exclusion Criteria- Pregnant women, teenage girls, hysterectomy patients, unsatisfactory smearand inadequate biopsies were excluded. Total of 50 cases were included in the study.

Methodology- Clinical details were obtained according to the structured proforma. The relevant clinical findings were collected by personal interview and examination of the patient. Pap smears were taken using Ayre's spatula from squamocolumnar junction. Material

\section{Original Research Article}

spread evenly on glass slide and fixed with cytofix containing 95\% ethyl alcohol and carbowax. Fixed smears received were stained with Pap stain and reported according to The Bethesda System.

Colposcopic examination was performed using Gold way SLC-2000 video colposcope. Normal saline was applied to the cervix to remove excess mucus. Green filter of colposcope was used to appreciate vascular pattern and $3 \%$ acetic acid applied to visualize the atypical transformation zone, following which biopsies were taken using punch biopsy forceps. Biopsy specimens received in $10 \%$ formalin fixative were routinely processed and sections stained with hematoxylin and eosin. Results were categorized according to WHO.

Statistical analysis- The data was coded and entered into Microsoft Excel spreadsheet. Analysis was done using SPSS version 15 (SPSS Inc. Chicago, IL, USA) Windows software program. Descriptive statistics included computation of percentages. For all tests, confidence level and level of significance were set at $95 \%$ and $5 \%$ respectively. Statistical analysis was carried out by calculating sensitivity, specificity, positive and negative predictive value for Pap smear and Colposcopy.

\section{Results}

Total of 50 cases were included in the study. Age range was from 20-70years with mean age of 36.4years. Majority of patients were in the age group of 21-30 years with 19 (38\%) cases followed by 31-40 years 17 (34\%), 41-50 years $8(16 \%), 51-60$ years $4(8 \%), 61-70$ years $1(2 \%)$ and $11-20$ years $1(2 \%)$.

Commonest clinical presentation was white discharge per vagina (WDPV) with 37 (74\%) cases, followed by irregular bleeding $6(21 \%)$, mass per vagina $3(6 \%)$, post-menopausal bleeding $2(4 \%)$ and pain abdomen with WDPV 2(4\%). In majority of patients' clinical status of cervix was cervical erosion with 27 (54\%) cases, followed by cervicitis 13 (26\%), cervical hypertrophy $7(14 \%)$, atrophy $2(4 \%)$ and cervical polyp $1(2 \%)$. Cytological diagnosis was inflammatory smear in $32(64 \%)$ cases followed by LSIL in $7(14 \%)$ Histological diagnosis was chronic nonspecific cervicitis among $33(66 \%)$ cases and CIN-19 (18\%) cases.

Table-1: Distribution of cases according to colposcopic findings.

\begin{tabular}{|c|c|c|}
\hline Colposcopic Findings & Number of cases & Percentage \\
\hline Acetowhite area & 20 & 40.0 \\
\hline Punctation & 06 & 12.0 \\
\hline Mosaicism & 02 & 04.0 \\
\hline Surface irregularity & 02 & 04.0 \\
\hline Atypical vessels & 02 & 04.0 \\
\hline Multiple abnormal colposcopic findings & 18 & 36.0 \\
\hline Total & 50 & 100 \\
\hline
\end{tabular}




\section{Original Research Article}

Commonest colposcopic finding was acetowhite area with 20 (40\%) cases, followed by multiple abnormal colposcopic finding $18(36 \%)$ [Table 1].

Table-2: Distribution of colposcopic diagnosis.

\begin{tabular}{|c|c|c|}
\hline Colposcopic Diagnosis & Number & Percentage \\
\hline Normal & 03 & 06.0 \\
\hline Inflammatory & 27 & 54.0 \\
\hline CIN1 & 12 & 24.0 \\
\hline CIN2 & 05 & 10.0 \\
\hline CIN3 & 01 & 02.0 \\
\hline Invasive carcinoma & 02 & 04.0 \\
\hline Total & 50 & 100 \\
\hline
\end{tabular}

Colposcopic diagnosis was inflammatory in 27 (54\%) cases followed by cervical intraepithelial neoplasia (CIN) 1 in 12 (24\%) patients [Table 2].

In correlation between cytology and biopsy, sensitivity of cytology was $82.3 \%$, specificity $96.9 \%$, false negative rate of $17.6 \%$, false positive rate of $3 \%$ and accuracy of $92 \%$.

In correlation between colposcopy and biopsy, sensitivity of colposcopy was $94.1 \%$, specificity $87.8 \%$, false negative rate of $6.25 \%$, false positive rate of $12.1 \%$ and accuracy of $90 \%$.

\section{Discussion}

Cancer of cervix is the fourth most common cancer in women, with an estimated 528,000 new cases in 2012 . Large majority (around $85 \%$ ) of the global burden occurs in the less developed regions, where it accounts for almost $12 \%$ of all female cancers.

Almost nine out of ten $(87 \%)$ cervical cancer deaths occur in the less developed regions [1]. In the present study, the age of patients ranged from 20 to 70 years with the mean age of 36.4 years, which is comparable to study by Joshi $C$ et al where the age range was 20 to 65 years, Similar distribution of patients has been observed in other studies also [5-7]. White discharge per vagina was the most common symptom with $37(74 \%)$ cases, which was comparable to studies done by Chaudhary $\mathrm{RD}$ et al and Bhalerao A et al $[8,9]$

In the present study, clinical status of cervix in majority of patients was cervical erosion with $27(54 \%)$ cases. Similar findings were observedin the studies performed by Chaudhary RD et al and Bhalerao A et al, where majority were cervical erosion with $173(86 \%)$, $156(78 \%)$ cases respectively[8,9].Most common colposcopic finding was acetowhite area with $20(40 \%)$ cases, similarly reported by Joshi $\mathrm{C}$ et al and Krishnegowda et al[10,11].
In the present study, on pap smear examination, squamous intraepithelial lesion of all grades were seen in $13(26 \%)$ cases [LSIL in $7(14 \%)$, HSIL in $6(12 \%)$ ] and SCC in 2(4\%), comparable to other studies [10,11].

Colposcopy was non-neoplastic in $30(60 \%)$ cases, CIN of all grades were seen in $18(36 \%)$ cases [CIN1 in $12(24 \%), \mathrm{CIN} 2$ in $5(10 \%), \mathrm{CIN} 3$ in $1(2 \%)$ ] and $2(4 \%)$ were invasive carcinoma. Seshadri L et al study showed CIN of all grades in 101(43.3\%) cases, $14(6.1 \%)$ invasive carcinoma and non- neoplastic in $118(50.6 \%)$ cases[12]. In the present study Histopathological diagnosis of cervicitis was seen $33(66 \%)$ cases, CIN of all grades in $15(30 \%)$ cases [CIN1 in $9(18 \%)$, CIN2 in $5(10 \%)$, CIN3 in $1(2 \%)$ ] and SCC $2(4 \%)$ comparable to study by Seshadri L et al[12].

Sensitivity of pap smear in the present study was $82.3 \%$, specificity was $96.9 \%$ and diagnostic accuracy was $92 \%$ which is comparable to study by Maziah et al $(90 \%)$, Bhatla et al $(89 \%)$. It was seen that the positive predictive value of Pap smear was highest for HSIL and malignancy.

This is similar to study by Naik et al[13-15]. Specificity of the pap smear was also comparable with studies by 


\section{Original Research Article}

Al Alwan et al 2001 (98.4\%),[16] Randomir et al 2005 (88\%),[17] and Mojgan et al 2011 (93\%)[18].

Sensitivity of colposcopy was $94.1 \%$ and specificity was $87.8 \%$. The accuracy of colposcopy in the present study was $90 \%$, which is in parallel to the findings of Maziah et al (94\%) and Ashmita et al (86.54\%)[14-16].

Cytology and colposcopy showed $100 \%$ correlation for high grade lesions. High false negative rate on cytology was seen in cases of inflammatory smear and high false positive rate on colposcopy in cases of CIN1, implying the importance of repeat smear and follow-up in these cases.

\section{Conclusion}

High sensitivity in colposcopy as compared to cytology and high specificity in cytology as compared to colposcopy emphasizes the need for pairing these methods to achieve better results.

Advantage of this study to existing knowledge- When properly used, colposcopy complements cytology by accurately defining the most suspicious area of the cervix for taking biopsy and there by increases the diagnostic accuracy.

Funding: Nil, Conflict of interest: None initiated, Permission from IRB: Yes

\section{References}

1. Ferlay J, Soerjomataram I, Dikshit R, EserS, Mathers C, Rebelo M, Parkin DM, Forman D, Bray F. Cancer incidence and mortality worldwide: sources, methods and major patterns in GLOBOCAN 2012. Int J Cancer. 2015 Mar 1;136(5):E359-86. doi: 10.1002/ijc.29210. Epub 2014 Oct 9.

2. Holowaty P, Miller AB, Rohan T, To T. Natural history of dysplasia of the uterine cervix. J Natl Cancer Inst. 1999 Feb 3;91(3):252-8.

3. Nanda K, McCrory DC, Myers ER, Bastian LA, Hasselblad V, Hickey JD, Matchar DB. Accuracy of the Papanicolaou test in screening for and follow-up of cervical cytologic abnormalities: a systematic review. Ann Intern Med. 2000 May 16;132(10):810-9.

4. Kholi B, Arya BS. Comparison of Pap smear and colposcopy in detection premalignant lesions of cervix. J South Asian Fed Menopause Soc 2014; 2:5-8.
5. Joshi C, Kujur P, Thakur N. Correlation of Pap Smear and Colposcopy in Relation to Histopathological Findings in Detection of Premalignant Lesions of Cervix in A Tertiary Care Centre. Int J Sci Stud 2015; 3:55-60.

6. Pimple SA, Amin G, Goswami S, Shastri SS. Evaluation of colposcopy vs cytology as secondary test to triage women found positive on visual inspection test. Indian J Cancer. 2010 Jul-Sep; 47 (3): 308-13. doi: 10. 4103/0019-509X.64726.

7. Boicea A, Pătraşcu A, Surlin V, Iliescu D, Schenker M, Chiuţu L. Correlations between colposcopy and histologicresults from colposcopically directed biopsy in cervical precance rouslesions. Rom J Morphol Embryol. 2012; 53 (3 Suppl): 735-41.

8. Chaudhary RD, Inamdar SA, Hariharan C. Correlation of diagnostic efficacy of unhealthy cervix by cytology, colposcopy and histopathology in women of rural areas. Int J Reprod Contracept Obstet Gynecol 2014; 3:213-218.

9. Bhalerao A, Kulkarni S, Ghike S, Kawthalkar A, Joshi S. Correlation of pap smear, colposcopy and histopathology in women with unhealthy cervix. J South Asian Feder Obst Gynae 2012; 4:9798 .

10. Joshi C, Kujur P, Thakur N. Correlation of Pap Smear and Colposcopy in Relation to Histopathological Findings in Detection of Premalignant Lesions of Cervix in A Tertiary Care Centre. Int J Sci Stud 2015; 3:55-60.

11. Krishnegowda S, Veena MS. Efficacy of coiposcopy technique with Pap smear and histology in screening of cervical lesions. Int J Repord Contracept ObstetGynecol 2014; 3:696-702.

12. Seshadri L, Jairaj P, Krishnaswami H. Colposcopy in the diagnosis of cervical neoplasia. Indian J Cancer. 1990 Sep;27(3):180-6.

13. Bhatla N, Mukhopadhyay A, Kriplani A, Pandey RM, Gravitt PE, Shah KV, Iyer VK, Verma K. Evaluation of adjunctive tests for cervical cancer screening in low resource settings. Indian $\mathrm{J}$ Cancer. 2007 Apr-Jun;44(2):51-5. 


\section{Original Research Article}

14. Maziah AM, Sharifah NA, Yahya A. Comparative study of cytologic and colposcopic findings in preclinical cervical cancer. Malays J Pathol 1991; 13:105-108.

15. Naik R, Minj MM, Panda R et al. Cytohistological correlation and accuracy of the Pap smear test in diagnosis of cervical lesions: a hospital based crosssectional study from Odisha, India. Medical Science $2015 ; 3: 242-249$.

16. Ashmita D, Shakuntala PN, Rao SR, et al. Comparison and Correlation of Pap smear, Colposcopy and Histopathology in Symptomatic Women and Suspicious Looking Cervix in a Tertiary Hospital Care Centre. Int J Health Sci Res 2013; 3:50-59.
17. Alwan A. Colposcopy, cervical cytology and human papillomavirus detection as screening tools for cervical cancer. Eastern Mediterranean Health Journal 2001;7:100-105.

18. Živadinović R, Radović M, Lilić V, Petrić S. Grading the severity of preinvasive changes of the uterine cervix by colposcopy and exfoliating cytology. FactaUniversitatis Series: Medicine and Biology 2005;12(1):55-59.

19. Karimi Zarchi M, Binesh F, Kazemi Z, Teimoori S, Soltani HR, Chiti Z. Value of colposcopy in the early diagnosis of cervical cancer in patients with abnormalpap smears at ShahidSadoughihospital, Yazd.Asian Pac J Cancer Prev. 2011;12(12):3439-41.

\section{How to cite this article?}

Upadhyay J, Garg S. Correlation of Pap smear and colposcopic finding with directed biopsy in detection of cervical neoplasm. Pathology Update:Trop J Path Micro 2017;3(4):396-400.doi:10.17511/jopm.2017.i4.06. 